\title{
A Non-Invasive Method for Detecting the Metabolic Stress Response in Rodents: Characterization and Disruption of the Circadian Corticosterone Rhythm
}

\author{
P. K. THANOS ${ }^{1-3}$, S. A. CAVIGELLI ${ }^{4}$, M. MICHAELIDES ${ }^{1-3^{*}}$, D. M. OLVET ${ }^{1,2^{*}}$, \\ U. PATEL ${ }^{1,3}$, M. N. DIEP ${ }^{4}$, N. D. VOLKOW ${ }^{3}$ \\ * These authors contributed equally to the study.
}

${ }^{1}$ Behavioral Neuropharmacology and Neuroimaging Laboratory, Department of Medicine, Brookhaven National Laboratory, ${ }^{2}$ Department of Psychology, SUNY Stony Brook, ${ }^{3}$ Laboratory of Neuroimaging, NIAAA, NIH, Department of Health and Human Services, Bethesda, MD, ${ }^{4}$ Department of Biobehavioral Health, Pennsylvania State University, University Park, PA, USA

Received October 23, 2007

Accepted January 18, 2008

On-line April 1, 2008

\begin{abstract}
Summary
Plasma corticosterone (CORT) measures are a common procedure to detect stress responses in rodents. However, the procedure is invasive and can influence CORT levels, making it less than ideal for monitoring CORT circadian rhythms. In the current paper, we examined the applicability of a non-invasive fecal CORT metabolite measure to assess the circadian rhythm. We compared fecal CORT metabolite levels to circulating CORT levels, and analyzed change in the fecal circadian rhythm following an acute stressor (i.e. blood sampling by tail veil catheter). Fecal and blood samples were collected from male adolescent rats and analyzed for CORT metabolites and circulating CORT respectively. Fecal samples were collected hourly for $24 \mathrm{~h}$ before and after blood draw. On average, peak fecal CORT metabolite values occurred 7-9 $\mathrm{h}$ after the plasma CORT peak and time-matched fecal CORT values were well correlated with plasma CORT. As a result of the rapid blood draw, fecal production and CORT levels were altered the next day. These results indicate fecal CORT metabolite measures can be used to assess conditions that disrupt the circadian CORT rhythm, and provide a method to measure long-term changes in CORT production. This can benefit research that requires longterm glucocorticoid assessment (e.g. stress mechanisms underlying health).
\end{abstract}

\section{Key words}

Corticosterone • Stress • Fecal steroid metabolites • Circadian rhythm

\section{Corresponding author}

P. K. Thanos, Brookhaven National Laboratory, Medical Department, Building 490, Upton, NY 11973, USA. Fax: (631) 344-5311. E-mail: thanos@bnl.gov

\section{Introduction}

Alterations in the glucocorticoid (CORT) circadian rhythm have been associated with mood disorders and drug addiction. Depressed patients exhibit changes in the CORT circadian rhythm, including a reduced quiescent time for secretion and phase advancement of the rhythm (Deuschle et al. 1997). Acute and chronic stress increases one's risk of alcohol and drug abuse and susceptibility for drug relapse (for review see Sinha 2001), and cocaine self-administration has been shown to alter the CORT circadian rhythm (Mantsch et al. 2003). Animal models provide a method to systematically examine stress mechanisms underlying mental health and drug abuse (e.g. Piazza and Le Moal 1998). Therefore, given the importance of the CORT circadian rhythm, developing techniques to measure this rhythm in laboratory animals provides an ideal method to further understand stress physiology underlying mood disorders and addictive behaviors. In the current study, we examined the applicability of a non-invasive fecal corticosterone metabolite measure to assess alterations in the normal glucocorticoid circadian rhythm in rats. It is

PHYSIOLOGICAL RESEARCH • ISSN 0862-8408 (print) • ISSN 1802-9973 (online)

(c) 2009 Institute of Physiology v.v.i., Academy of Sciences of the Czech Republic, Prague, Czech Republic

Fax +420 241062 164, e-mail: physres@biomed.cas.cz, www.biomed.cas.cz/physiolres 
important to note that corticosterone is the predominant glucocorticoid in many laboratory animals, while cortisol is the predominant glucocorticoid in humans.

Unfortunately, measuring the CORT rhythm in animal models of these stress-related conditions can be limited by methodological factors that introduce unwanted and uncontrolled stressors (for review see Mormede et al. 2007). Blood sampling involves procedures such as physical restraint that significantly elevate CORT levels (Pace and Spencer 2005). Even the simple acts of husbandry-related disturbances, transport, handling, and novel caging can induce CORT elevations in rodents (Cavigelli et al. 2006, Fluttert et al. 2000, Gartner et al. 1980, Tuli et al. 1995). Thus, if one is interested in assessing rodents' physiological stress response to an experimentally-imposed stressor (e.g. behavioral tests, environmental or physiological manipulations, etc.), it is difficult to track this response for long periods without influencing it with measurement procedures. Minimally invasive or non-invasive sample collection methods for CORT assessment provide an appealing method for assessing hypothalamo-pituitaryadrenal (HPA) axis function without altering it in the process.

One method used for repeated blood sampling is jugular cannulation. This method is beneficial because it allows for multiple blood samples to be obtained as well as large quantities of blood, but it can include several drawbacks. It is difficult to keep the catheter patent and the catheter itself can serve as a source of irritation and infection (Sadoyama and Gontijo Filho, 2003, Yang et al. 2005). Jugular cannulation also limits the animal's mobility and can be viewed as a stressor since it disrupts CORT patterns (Royo et al. 2004). Similarly, factors associated with cannula surgery, such as anesthesia, blood loss, and pain, have been shown to disrupt basal CORT stress levels (Haemisch et al. 1999, Tuli et al. 1995, Vahl et al. 2005, Wright-Williams et al. 2006). Finally, blood samples provide a momentary measure of circulating hormone levels which are highly variable due to the pulsatile nature of steroid release (Windle et al. 1998).

Other blood collection methods have been utilized. Both tail vein nick and tail-snip allow for the acquisition of small blood samples. It has been shown that these methods do not induce a stress response when samples are collected within $3 \mathrm{~min}$, but these methods are invasive (Fluttert et al. 2000, Vahl et al. 2005). Terminal trunk blood collection can also be used, but anesthetics such as $\mathrm{CO}_{2}$ and sodium pentobarbital used prior to decapitation cause significant increases in CORT levels (Vahl et al. 2005). Even something as simple as an intraperitoneal (IP) saline injection can affect CORT (Harris et al. 2004).

In this study the Minimally Invasive Rapid Blood (MIRB) sampling method was used; in which animals are briefly restrained (not anesthetized), while a small catheter is placed into the tail vein to collect blood. MIRB has been used successfully for repeated blood sampling with Wistar rats. Results from 2-h repeated sampling indicated that CORT levels were significantly elevated $20 \mathrm{~min}$ following catheterization and then returned to baseline levels within $120 \mathrm{~min}$ (Haemisch et al. 1999).

Recently, a non-invasive method for CORT assessment has been developed. Rat fecal pellets contain high concentrations of CORT metabolites and these metabolites can be extracted and measured (Cavigelli et al. 2005). Previous research has shown that after an intraperitoneal injection of radioactive CORT Sprague Dawley rats excreted $80 \%$ of the recovered metabolites in feces (Bamberg et al. 2001). The circadian fecal CORT metabolite rhythm is delayed between 6-12 h compared to the circulating CORT rhythm as assessed with blood, but the temporal dynamics of the circadian rhythm are very similar (Cavigelli et al. 2005, Royo et al. 2004, Touma et al. 2003). Another consideration to be made is that plasma samples only measure single point concentrations, whereas fecal samples measure interval concentrations (Touma and Palme 2005). A single plasma value provides a momentary measure at the time of sampling, whereas a fecal measure provides an integrated measure of CORT production over hours. Fecal measures can provide a complement to acute blood sample CORT measures. For long-term CORT assessments, fecal pellets are ideal for small rodents because they have limited blood volumes and they defecate several pellets every 1-2 h (Kishibayashi et al. 1995). One limitation of these methods is that the relationship between circulating CORT and excreted CORT metabolite levels is as yet unknown.

In the present study, we assessed and compared the circadian rhythm of plasma and fecal CORT. While previous findings suggested that rapid tail vein blood sampling (MIRB) elevated circulating CORT levels for a short period (i.e. less than $2 \mathrm{~h}$ ), we further examined the stressful nature of this procedure by measuring fecal CORT levels the day after MIRB. MIRB sampling 
involves handling, physical restraint and blood loss - all of which may stimulate a physiological stress response. We also compared plasma levels of CORT with fecal CORT metabolite levels within individual animals to determine if the fecal CORT measure provided an accurate assessment of circulating CORT. In addition, we collected circadian locomotor activity as verification of the normal circadian rhythm prior to blood sampling. We hypothesized that fecal CORT metabolite levels would be positively correlated with plasma CORT levels and that a minimally-invasive blood sampling procedure may illicit a longer stress response than previously expected, which would be detectable in the fecal CORT metabolite measures.

\section{Methods}

Animals

Twenty four male (4-6 weeks old, mean body weight $226.5 \pm 6.13 \mathrm{~g})$ Sprague Dawley Rats (Charles River) were individually housed in a $12: 12 \mathrm{~h}$ reverse light/dark cycle with the dark phase beginning at 08:00 h. Temperature in the animal room was maintained between $22 \pm 1{ }^{\circ} \mathrm{C}$ and humidity was between $40-60 \%$. Rats were fed daily at 13:30 and were kept on a restricted diet $(5 \mathrm{~g}$ food/100 $\mathrm{g}$ body weight). All animals lived in individual metabolic type cages (Hoeltge Inc, Ohio, USA; $23 \mathrm{~cm}$ depth $\times 19 \mathrm{~cm}$ width $\times 19 \mathrm{~cm}$ height) with wire bottoms through which feces dropped and were collected hourly and immediately frozen. Animals were housed in theses cages for 1 week prior to the study to allow for habituation and they were each handled 5 min daily. Animals were run in two batches, two weeks apart, due to the limited number of locomotor arenas available (Fig. 1). This work was approved by the Institutional Animal Care and Use Committee (IACUC) of Brookhaven National Laboratory in accordance with the guidelines established by the NIH in The Guide For Care and Use of Laboratory Animals.

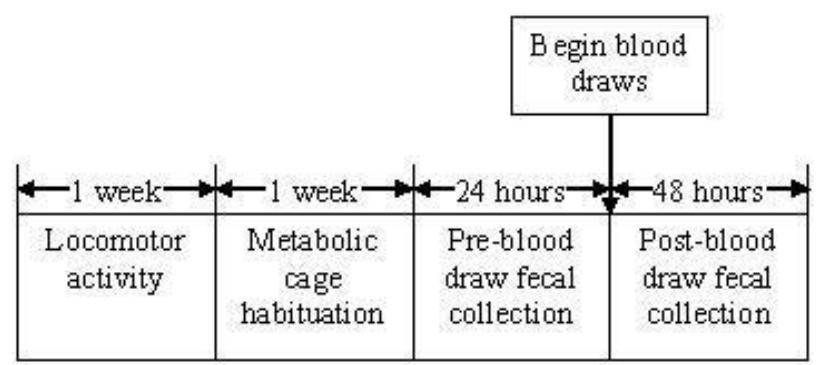

Fig. 1. Study time-line.

\section{Locomotor activity}

As an additional measure of normal circadian rhythm prior to blood collection, rats were placed in an Optical Sensor Plexiglas arena (Minimitter Corporation, Oregon, USA) with dimensions identical to their home cages. Locomotor activity was recorded from undisturbed animals $24 \mathrm{~h} /$ day for seven consecutive days and during this time temperature and humidity levels were consistently monitored to meet IACUC requirements. Optical beam breaks were recorded as the sum of all beam breaks every minute and the total number of beam breaks/hour was reported within a $24 \mathrm{~h}$ circadian clock. In order to limit habituation effects in our data, we analyzed the average of the sixth and seventh day values and did not take the earlier daily data into consideration.

\section{Fecal sample collection}

Fecal samples were collected hourly for $24 \mathrm{~h}$ before and after blood draw to characterize the circadian rhythm. Fecal samples that were contaminated with urine were not collected. Uncontaminated samples were collected and transferred to a zip lock bag, labeled, and immediately stored at $-80{ }^{\circ} \mathrm{C}$. A total of 397 fecal samples were collected across 24 rats.

\section{Blood sample collection}

Blood samples were obtained from three rats every $3 \mathrm{~h}$ starting at 08:00, therefore samples were collected at the following time-points: 08:00, 11:00, 14:00, 17:00, 20:00, 23:00, 02:00, and 05:00. The three rats within each time-point are referred to as a group for statistical analyses. Each animal was sampled only once by MIRB sampling via tail vein catheterization. Animals were restrained in a plastic cone (DecapiCone, MA, USA) and a 24 gauge catheter was inserted into the tail vein. The time required to sample blood from each animal did not exceed $3 \mathrm{~min}$. Between 400-500 $\mu \mathrm{l}$ of blood was collected in an EDTA $_{\mathrm{K}}$ and aprotinin-coated Multivette tube (Sarstedt, Germany) and centrifuged for $10 \mathrm{~min}$ at $4{ }^{\circ} \mathrm{C}$. Plasma was then pipetted into cryovials and immediately stored at $-80^{\circ} \mathrm{C}$ until analysis.

\section{Fecal corticoid extraction}

Fecal steroids were extracted using previously published methods (Cavigelli et al. 2005). Briefly, frozen samples were thawed, dried in a centrifugal evaporator, crushed into a dust-like material, and $0.2 \mathrm{~g}$ weighed into a $15 \mathrm{ml}$ centrifuge tube. Ethanol (100\%) was added to each sample then boiled in a water bath for $20 \mathrm{~min}$. After 
removal from the bath, tubes were centrifuged $15 \mathrm{~min}$ and supernatant poured into a glass tube. Ethanol was added to the fecal sample, vortexed $1 \mathrm{~min}$, re-centrifuged for $15 \mathrm{~min}$, and the supernatant added to the previous $10 \mathrm{ml}$ of extract. Supernatants were evaporated under air, reconstituted with methanol and stored at $-80{ }^{\circ} \mathrm{C}$ until assay. Fecal CORT metabolite values are expressed as $\mathrm{ng} / \mathrm{g}$ of dry fecal weight.

\section{Radioimmunoassay (RIA)}

A commercially available $\left[{ }^{125} \mathrm{I}\right] \mathrm{RIA}$ (MP Biomedical, Solon, OH, USA) for rat and mouse serum/plasma CORT was used to quantify fecal corticoids. Cross-reactivity of this RIA antibody with fecal CORT metabolites has not been tested by the company. However, in previous work, we have determined that the above extraction method and RIA combination detects several CORT metabolites (11-dehydrocorticosterone, tetrahydrocorticosterone, 21-hydroxy-5 $\beta$-pregnane-3, 11, 20-trione) (Cavigelli et al. 2005). To ensure antibody binding along the linear portion of the standard curve (20 and $80 \%$ binding), fecal extracts were diluted 1:40 (or more) with RIA kit diluent. Samples were assayed in duplicate and re-analyzed if the coefficient of variance exceeded $10 \%$. Two control samples were analyzed in every assay (a 'low' pool at approximately $60 \%$ binding, and a 'high' pool at approximately $25 \%$ binding). Based on repeated analysis of these samples, intra- and inter-assay coefficients of variance were $8.42 \%(n=7)$ and $17.56 \%(n=6)$ for the low pool and $7.88 \%(n=7)$ and $8.82 \%(n=7)$ for the high pool.

\section{Statistical analyses}

Data are presented as mean \pm S.E.M. for each sampling interval. Time is represented on a scale from 0 to 23 , with 0 equal to $8: 00 \mathrm{~h}$ and 23 equal to 7:00 h. Times are represented in accordance with this scale (i.e. time 0 is expressed as $\mathrm{T}=0$ ). Fecal CORT metabolite levels were log transformed for analysis; figures represent raw data. We used a one-way analysis of variance (ANOVA) and two-way (group $\mathrm{x}$ time-point) repeated measures ANOVA to determine if fecal CORT metabolite levels, fecal weight, and/or mean defecation intervals were different between the pre- and post-blood sampling days. Because each rat did not defecate at each hourly interval, we had several missing samples. To minimize this effect, we repeated the ANOVA using fecal CORT metabolite values averaged over 3-h intervals (e.g.
5-7 h, 8-10 h, 11-13 h, etc.). We compared individual fecal CORT metabolite values to their plasma CORT values in the following way. To control for the daily circadian rhythm and sampling order, plasma CORT values were standardized to the time of day and order of sample collection using regression analyses. Individual residual plasma CORT values were compared to fecal CORT metabolite values collected within a similar part of the day. This avoided samples from the highly variable 'peak' of 7-12 h and controlled for the circadian rhythm in fecal CORT. We used correlation analyses to compare residual plasma CORT to fecal CORT metabolite values. Rats with plasma CORT values greater than 1.5 SD from the mean were removed as outliers. Possible disruption of fecal output was identified by the time it took an animal to first defecate after blood draw. This value was compared to the time to defecate after a matched time point on the day prior to the blood draw procedure (e.g. if the blood draw occurred at 08:00, the time to defecate post-blood draw was compared to the time to defecate after 08:00 the day before the blood collection procedure). We also compared the circadian rhythm in fecal weight for the day prior to and following the blood collection procedure using repeated-measures ANOVA.

\section{Results}

\section{Locomotor activity}

One-way ANOVA revealed that there was a significant difference in the total number of beam breaks between the light and dark cycle $[F(1,526)=204.86$; $\mathrm{p}<0.001]$. Figure 2 shows the circadian locomotor activity rhythm which serves as a confirmation that the animals in this study were entrained to a $12: 12 \mathrm{~h}$ D:L cycle.

\section{CORT circadian rhythm}

Plasma and fecal CORT circadian rhythms the day prior to the blood collection procedure are shown in Figure 3. These values from the day prior to the blood collection procedure are referred to as 'pre-blood draw' levels. There was a significant (one-way ANOVA) difference in plasma CORT levels between dark $(\mathrm{T}=$ $0-11)$ and light cycle $(\mathrm{T}=12-23),[\mathrm{F}(1,22)=8.80$; $\mathrm{p}<0.01]$. There was also a significant difference in preblood draw fecal CORT metabolite levels between the fecal dark $(\mathrm{T}=9-20)$ and light $(\mathrm{T}=21-8)$ cycle $[\mathrm{F}(1,211)$ $=20.7 ; \mathrm{p}<0.001)$ and post-blood draw fecal CORT metabolite levels between the fecal dark $(T=9-20)$ and 

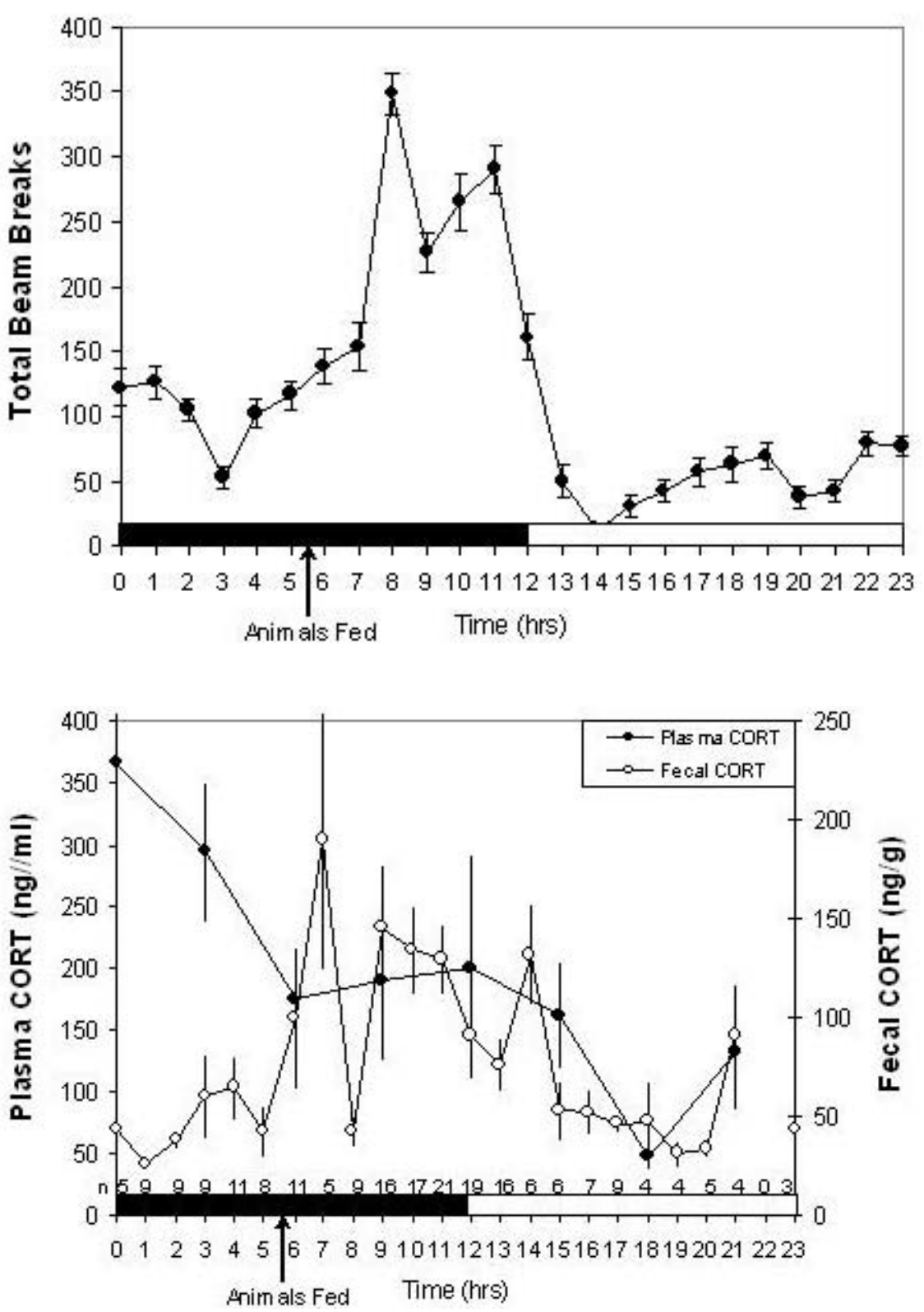

Fig. 2. Circadian rhythm locomotor activity $(n=22)$ represented by total beam breaks. Dark shading along the $x-$ axis represents the dark phase of the light cycle, white shading represents the light phase. Animals were fed at 13:30 h. Data are presented as means \pm S.E.M.

Fig. 3. Corticosterone (CORT) circadian rhythm in plasma ( $n=3 /$ time-point) and feces. $\mathrm{N}$ for feces are listed on the $\mathrm{X}$-axis for each time-point. Plasma CORT is represented by dark circles and fecal CORT metabolites are represented by open circles. Dark shading along the $x$ axis represents the dark phase of the light cycle, white represents the light phase. Animals were fed at 13:30 h. Data are presented as means \pm S.E.M. light $(\mathrm{T}=21-8)$ cycle $[\mathrm{F}(1,138)=11.1 ; \mathrm{p}<0.001]$. Fecal CORT metabolite values peaked $7 \mathrm{~h}$ after plasma CORT values. This peak was based on values from only 5 males, one of which had a particularly elevated fecal CORT metabolite value (2.5 times higher than the other males). Therefore, it may be more accurate to estimate the fecal circadian peaked between 7-9 $\mathrm{h}$ following the circulating CORT peak (Fig. 3).

\section{Plasma vs. fecal CORT}

Time-matched fecal CORT metabolite values were closely related to residual plasma CORT values $\left(\mathrm{r}_{19}=0.86, \mathrm{p}<0.0001\right)$ (Fig. 4), indicating that fecal CORT metabolite measures provide an accurate estimate of individual circulating glucocorticoid levels.
Pre- vs. post-blood draw fecal CORT levels

There was a significant difference between preand post-blood draw fecal CORT metabolite levels hourly $[2 \times 1$ mixed ANOVA; $F(1,24)=7.0 ; \mathrm{p}<0.05]$ and when samples were averaged every $3 \mathrm{~h}$ [Fig. 5; 2x1 mixed ANOVA, $\mathrm{F}(1,32)=8.16 ; \mathrm{p}<0.01]$. Using hourly data, there was no significant interactions between day and group $[\mathrm{F}(1,24)=1.9, \mathrm{p}>0.05]$, and between day, group and time-point $[\mathrm{F}(20,24)=1.6, \mathrm{p}>0.05]$, but there was a significant interaction between day and time-point $[\mathrm{F}(7,24)=3.9, \mathrm{p}<0.01]$. Using the 3 -h averaged data, there was no significant interactions between day (pre- and post-blood draw fecal CORT metabolite levels) and group $[\mathrm{F}(7,32)=0.53 ; \mathrm{p}>0.05]$, between day and time-point $[\mathrm{F}(7,32)=0.55 ; \mathrm{p}>0.05]$, or between day, group and time-point $[\mathrm{F}(26,32)=-0.49 ; \mathrm{p}>0.05]$. Post- 


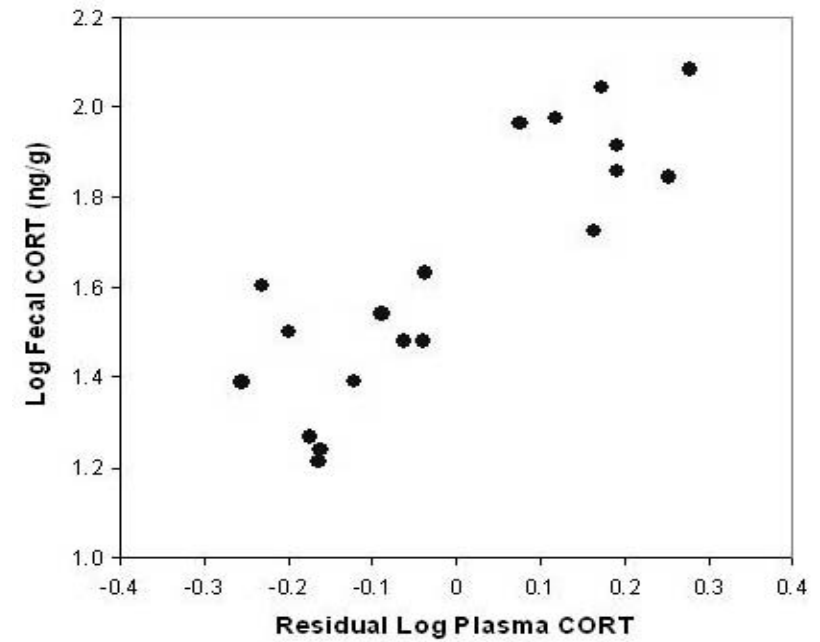

Fig. 4. Fecal CORT metabolite values for individual males compared to residual plasma CORT.

hoc comparisons were done using Student's paired t-test. Pre- and post-blood draw fecal CORT metabolite level differences were significantly greater at $\mathrm{T}=9-11$ (circadian peak) than at the following time points: $\mathrm{T}=$ $0-2(0.43 \pm 0.10 ; \mathrm{p}<0.01), \mathrm{T}=6-8(0.33 \pm 0.08 ; \mathrm{p}<0.01)$, $\mathrm{T}=15-17(0.39 \pm 0.08 ; \mathrm{p}<0.001)$, and $\mathrm{T}=18-20(0.51 \pm$ $0.13 ; \mathrm{p}<0.01)$. Figure 5 shows pre- and post-blood draw fecal CORT metabolite values at 3-h time intervals. Postblood fecal CORT metabolite levels were significantly greater than pre-blood levels at $\mathrm{T}=0-2(\mathrm{p}<0.01)$ and $\mathrm{T}=$ $12-14(\mathrm{p}<0.01)$.

\section{Disruption of fecal output}

There was a significant difference between the interval required to defecate post-blood draw $(7 \mathrm{~h} 12 \mathrm{~min}$ $\pm 42 \mathrm{~min}$ ) and the interval at a matched time pre-blood draw (2 h 55 min \pm 32 min; Fig. 6; 2x1 mixed ANOVA; $\mathrm{F}(1,16)=42.58 ; \mathrm{p}<0.001]$. There were no significant differences between pre- and post-blood draw fecal weight when using hourly weights $[2 \times 1$ mixed ANOVA; $\mathrm{F}(1,30)=0.23 ; \mathrm{p}>0.05]$ or 3 -h averaged weights $[2 \mathrm{x} 1$ mixed ANOVA; $\mathrm{F}(1,37)=0.96 ; \mathrm{p}>0.05]$. However, the circadian rhythm of fecal weight changed from pre- to post-blood draw (day $\mathrm{x}$ time-point interaction with 3-hr averaged weights $[\mathrm{F}(7,37)=2.51 ; \mathrm{p}<0.05])$. This is additional evidence of the stressful nature of the MIRB blood draw.

\section{Discussion}

We show that fecal CORT metabolite levels can be used as a non-invasive estimate of circadian glucocorticoid production in rats. The fecal CORT metabolite circadian rhythm was time-shifted from the plasma CORT rhythm by approximately 7-9 $\mathrm{h}-\mathrm{a}$ shift consistent with prior studies (Cavigelli et al. 2005, Royo et al. 2004, Touma et al. 2003). Fecal corticoid measures reflect circulating CORT levels. Thus, fecal output rhythm and fecal CORT metabolite levels were altered by a minimally invasive blood sampling procedure.

Indeed, the minimally-invasive rapid blood (MIRB) sampling led to a significant alteration in the fecal CORT metabolite rhythm one day later (Fig. 5). Statistically, there was no interaction of day (pre- vs. post-blood draw) and group (specific time of each blood draw), which confirmed that stressful effects of blood draw were not dependent on the time of blood draw. This is important because many studies utilize stressors at different times of the light/dark cycle and studies often use unpredictable stressors that occur randomly over the course of the week.

The relationship between plasma CORT and fecal CORT metabolites was linear and strong. This is the first evidence that individual fecal CORT metabolite levels reflect individual circulating levels in laboratory rats. Further work will be necessary to identify appropriate analysis strategies to account for uncontrollable missing samples. However, the current results are promising in terms of the functional significance of non-invasive fecal CORT metabolite measures. These results indicate that we can safely assume individuals with high fecal CORT output will also have high circulating CORT levels. Although blood measures are more beneficial for studies of short-term changes in CORT secretion, fecal CORT metabolite measures provide a nice complement to these traditional measures when researchers want to understand long-term CORT profiles in laboratory rodents.

Confirmation of a stress response is seen in the disruption of post-blood draw fecal output. MIRB sampling caused a $285 \pm 64 \%$ increase in the time to defecate post-blood draw (compared to the same time the previous day with no blood draw) and also altered the circadian rhythm in fecal output. This is a significant behavioral and metabolic finding, and yet it was also detrimental to our study in that there were limited fecal samples for analysis post-blood draw. In order to maximize the number of samples we were able to collect, we averaged fecal CORT metabolite levels over a three hour period. This sampling interval was previously used in other studies (Cavigelli et al. 2005, Touma et al. 


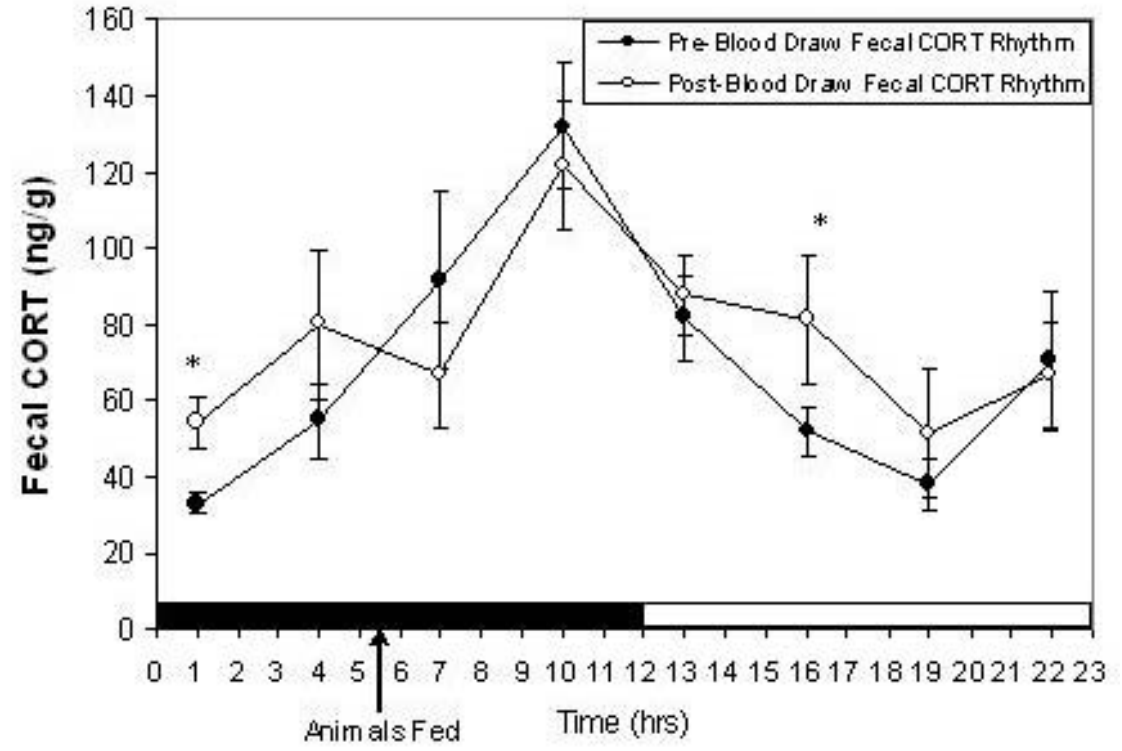

Fig. 5. Pre-blood draw (dark circles) and post-blood draw (open circles) fecal CORT metabolite levels averaged across all groups. Dark shading along the $x$-axis represents the dark phase of the light cycle, white represents the light phase. Animals were fed at 13:30h. Data are represented as means \pm S.E.M.

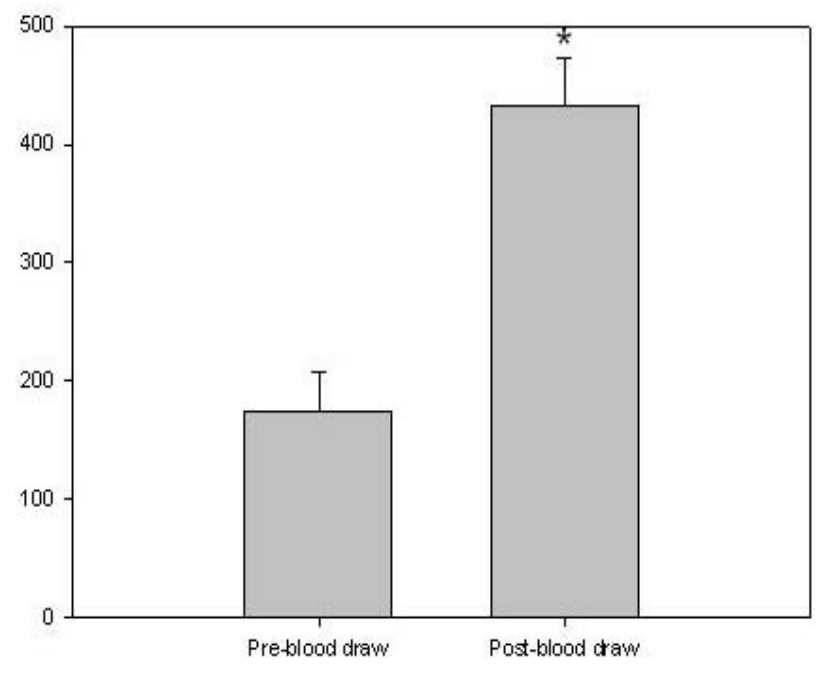

Fig. 6. Time ( $\mathrm{min}$ ) to first fecal output pre- and post-blood draw. Data is represented as means \pm S.E.M.

2003), as a reliable estimate of CORT levels and was more practical than hourly sampling.

The time of feeding is important to consider when evaluating circadian rhythms. Rats fed on an ad libitum schedule will typically eat at the start of the dark cycle and show a pronounced circadian rhythm in fecal output (weight). In the present study, this normally strong rhythm was disrupted by the minimally invasive rapid blood draw procedure that required removing a rat from it's home cage for no longer than $5 \mathrm{~min}$. These results are important to consider when conducting minimally invasive protocols; even seemingly small changes in the laboratory rat environment can lead to pronounced changes in physiology (Sharp et al. 2002).

There are additional facets of this study that may contribute to the stress response. We housed animals in metabolic cages starting one week prior to fecal collection. It has been shown that housing rats in metabolic cages for three days is a mild stressor (Eriksson et al. 2004). However, one week should provide sufficient time for the animals to habituate to the metabolic cages and our results on locomotor activity confirm this. Additionally, the animals were handled 5 min each, daily for two weeks prior to fecal collection to minimize any acute CORT response due to handling. This was meant to minimize stress levels when animals were taken out of their cage for the blood draw. Animals were also temporarily restrained (for 2-3 $\mathrm{min}$ ) in order to draw blood from the tail vein. We did not want to use anesthesia because it has been shown that anesthesia can have an impact on CORT levels (Vahl et al. 2005). Additionally, we drew blood samples under three minutes to avoid an acute increase in CORT. To collect blood samples we minimized as many stressful aspects of the procedure as possible, yet we still found significant alterations in fecal output and fecal CORT metabolite levels the following day. These results provide further support for the development of meaningful non-invasive CORT measures to assess CORT circadian rhythms.

In this study, we have shown that analyzing fecal CORT metabolites is a sensitive method to assess alterations in rodent CORT production. This procedure allows for detection of HPA axis disruption with a measure that does not involve the risk of inducing stress. One limitation of this procedure is that it may not be practical to measure acute CORT responses because short elevations in these circulating steroids may not be easy to 
detect in integrated fecal measures. However, this method provides a non-invasive alternative for chronic stress studies as it allows researchers to track CORT level changes over long periods of time.

Additionally, this method can be used in conjunction with behavior and in vivo brain imaging [using positron emission tomography (PET) or MRI] to look at brain regional activity in rodent studies of chronic stress. Changes in brain glucose utilization has been previously shown in rodent studies on stress (Brown et al. 1996, Duncan et al. 1993), but also in response to drugs some of which mimic the effects of stressors methylphenidate (Porrino and Lucignani 1987), cocaine (Macey et al. 2004, Porrino et al. 2002), alcohol (Williams-Hemby et al. 1996) and $\Delta 9$-tetrahydrocannabinol (Whitlow et al. 2002). Since stress- and drugrelated cues influence regional brain activity and play an important role in the high rates of relapse observed in alcohol and drug abuse it is critical that all these factors be examined within the same subject. Neuroimaging studies have thus begun to identify the CNS correlates of stress and drug cue-induced craving and these findings show considerable overlap in neural circuits involved in processing stress and drug cues with activity in the cortico-striatal limbic circuitry underlying both affective and reward processing.

In conclusion, the current study thus provides a non-invasive longitudinal method for assessing rodent CORT levels and its relationship to, the same animal's behavior and brain regional responses throughout the course of an experiment. Utilizing rodent fecal samples to obtain CORT metabolite measurements may benefit many lines of neuroscience research that requires the measurement of long-term glucocorticoid production.

\section{Conflict of Interest}

There is no conflict of interest.

\section{Acknowledgements}

This work was supported by the NIAAA Intramural Research Program (AA 11034 \& AA07574, AA07611) and by the U.S. Department of Energy under contract DE-AC02-98CH10886 and by internal funding from the Pennsylvania State University. We thank Vasilios Boronikolas, Carlos Bermeo, Roberto Ramalhete, Yu Fan Hwang, Yiannis Piyis, Rahila Habibi, Kelsie Haskins, Colleen Kovacsics, Cole Matteo, Krishna Mehta and Janey Quinn for their assistance with the sample collections and processing.

\section{References}

BAMBERG E, PALME R, MEINGASSNER JG: Excretion of corticosteroid metabolites in urine and faeces of rats. Lab Anim 35: 307-314, 2001.

BROWN LL, SIEGEL H, ETGEN AM: Global sex differences in stress-induced activation of cerebral metabolism revealed by 2-deoxyglucose autoradiography. Horm Behav 30: 611-617, 1996.

CAVIGELLI SA, MONFORT SL, WHITNEY TK, MECHREF YS, NOVOTNY M, MCCLINTOCK MK: Frequent serial fecal corticoid measures from rats reflect circadian and ovarian corticosterone rhythms. $J$ Endocrinol 184: 153-163, 2005.

CAVIGELLI SA, GUHAD FA, CEBALLOS RM, WHETZEL CA, NEVALAINEN T, LANG CM, KLEIN LC: Fecal corticoid metabolites in aged male and female rats after husbandry-related disturbances in the colony room. J Am Assoc Lab Anim Sci 45: 17-21, 2006.

DEUSCHLE M, SCHWEIGER U, WEBER B, GOTTHARDT U, KORNER A, SCHMIDER J, STANDHARDT H, LAMMERS CH, HEUSER I: Diurnal activity and pulsatility of the hypothalamus-pituitary-adrenal system in male depressed patients and healthy controls. J Clin Endocrinol Metab 82: 234-238, 1997.

DUNCAN GE, JOHNSON KB, BREESE GR: Topographic patterns of brain activity in response to swim stress: assessment by 2-deoxyglucose uptake and expression of Fos-like immunoreactivity. J Neurosci 13: 3932-3943, 1993.

ERIKSSON E, ROYO F, LYBERG K, CARLSSON HE, HAU J: Effect of metabolic cage housing on immunoglobulin A and corticosterone excretion in faeces and urine of young male rats. Exp Physiol 89: 427-433, 2004.

FLUTTERT M, DALM S, OITZL MS: A refined method for sequential blood sampling by tail incision in rats. Lab Anim 34: 372-378, 2000. 
GARTNER K, BUTTNER D, DOHLER K, FRIEDEL R, LINDENA J, TRAUTSCHOLD I: Stress response of rats to handling and experimental procedures. Lab Anim 14: 267-274, 1980.

HAEMISCH A, GUERRA G, FURKERT J: Adaptation of corticosterone-but not beta-endorphin-secretion to repeated blood sampling in rats. Lab Anim 33: 185-191, 1999.

HARRIS RB, GU H, MITCHELL TD, ENDALE L, RUSSO M, RYAN DH: Increased glucocorticoid response to a novel stress in rats that have been restrained. Physiol Behav 81: 557-568, 2004.

KISHIBAYASHI N, YOKOYAMA T, KARASAWA A: Enhancement of defecation and distal colonic motor activity by KW-5092, a novel gastroprokinetic agent, in rats. Arch Int Pharmacodyn Ther 329: 295-306, 1995.

MACEY DJ, RICE WN, FREEDLAND CS, WHITLOW CT, PORRINO LJ: Patterns of functional activity associated with cocaine self-administration in the rat change over time. Psychopharmacology (Berl) 172: 384-392, 2004.

MANTSCH JR, YUFEROV V, MATHIEU-KIA AM, HO A, KREEK MJ: Neuroendocrine alterations in a high-dose, extended-access rat self-administration model of escalating cocaine use. Psychoneuroendocrinology 28: 836$862,2003$.

MORMEDE P, ANDANSON S, AUPERIN B, BEERDA B, GUEMENE D, MALMKVIST J, MANTECA X, MANTEUFFEL G, PRUNET P, VAN REENEN CG, RICHARD, S, VEISSIER I: Exploration of the hypothalamic-pituitary-adrenal function as a tool to evaluate animal welfare. Physiol Behav 92: 317-339, 2007.

PACE TW, SPENCER RL: Disruption of mineralocorticoid receptor function increases corticosterone responding to a mild, but not moderate, psychological stressor. Am J Physiol Endocrinol Metab 288: E1082-E1088, 2005.

PIAZZA PV, LE MOAL M: The role of stress in drug self-administration. Trends Pharmacol Sci 19: 67-74, 1998.

PORRINO LJ, LUCIGNANI G: Different patterns of local brain energy metabolism associated with high and low doses of methylphenidate. Relevance to its action in hyperactive children. Biol Psychiatry 22: 126-138, 1987.

PORRINO LJ, LYONS D, MILLER MD, SMITH HR, FRIEDMAN DP, DAUNAIS JB, NADER MA: Metabolic mapping of the effects of cocaine during the initial phases of self-administration in the nonhuman primate. J Neurosci 22: 7687-7694, 2002.

ROYO F, BJORK N, CARLSSON HE, MAYO S, HAU J: Impact of chronic catheterization and automated blood sampling (Accusampler) on serum corticosterone and fecal immunoreactive corticosterone metabolites and immunoglobulin A in male rats. $J$ Endocrinol 180: 145-153, 2004.

SADOYAMA G, GONTIJO FILHO PP: Comparison between the jugular and subclavian vein as insertion site for central venous catheters: microbiological aspects and risk factors for colonization and infection. Braz J Infect Dis 7: 142-148, 2003.

SHARP JL, ZAMMIT TG, AZAR TA, LAWSON, DM: Stress-like responses to common procedures in male rats housed alone or with other rats. Contemp Top Lab Anim Sci 41: 8-14, 2002.

SINHA R: How does stress increase risk of drug abuse and relapse? Psychopharmacology (Berl) 158: 343-359, 2001.

TOUMA C, PALME R: Measuring fecal glucocorticoid metabolites in mammals and birds: the importance of validation. Ann N Y Acad Sci 1046: 54-74, 2005.

TOUMA C, SACHSER N, MOSTL E, PALME R: Effects of sex and time of day on metabolism and excretion of corticosterone in urine and feces of mice. Gen Comp Endocrinol 130: 267-278, 2003.

TULI JS, SMITH JA, MORTON DB: Corticosterone, adrenal and spleen weight in mice after tail bleeding, and its effect on nearby animals. Lab Anim 29: 90-95, 1995.

VAHL TP, ULRICH-LAI YM, OSTRANDER MM, DOLGAS CM, ELFERS EE, SEELEY RJ, D'ALESSIO DA, HERMAN JP: Comparative analysis of ACTH and corticosterone sampling methods in rats. Am J Physiol 289: E823-E828, 2005.

WHITLOW CT, FREEDLAND CS, PORRINO LJ: Metabolic mapping of the time-dependent effects of $\Delta 9$-tetrahydrocannabinol administration in the rat. Psychopharmacology (Berl) 161: 129-136, 2002.

WILLIAMS-HEMBY L, GRANT KA, GATTO GJ, PORRINO LJ: Metabolic mapping of the effects of chronic voluntary ethanol consumption in rats. Pharmacol Biochem Behav 54: 415-423, 1996.

WINDLE RJ, WOOD SA, LIGHTMAN SL, INGRAM CD: The pulsatile characteristics of hypothalamo-pituitaryadrenal activity in female Lewis and Fischer 344 rats and its relationship to differential stress responses. Endocrinology 139: 4044-4052, 1998. 
WRIGHT-WILLIAMS SL, COURADE JP, RICHARDSON CA, ROUGHAN JV, FLECKNELL PA: Effects of vasectomy surgery and meloxicam treatment on faecal corticosterone levels and behaviour in two strains of laboratory mouse. Pain 130: 108-118, 2006.

YANG J, MAAREK JM, HOLSCHNEIDER DP: In vivo quantitative assessment of catheter patency in rats. Lab Anim 39: 259-268, 2005. 